\title{
Continuous Representations of Speed by Striatal Medium Spiny Neurons
}

\author{
DWambura C. Fobbs, ${ }^{1}$ Sebastiano Bariselli , ${ }^{1}$ Julia A. Licholai, ${ }^{1}$ Nanami L. Miyazaki, ${ }^{1}$ Bridget A. Matikainen-Ankney, ${ }^{1}$ \\ Meaghan C. Creed, ${ }^{2}$ and ${ }^{\circ}$ Alexxai V. Kravitz ${ }^{1,2}$ \\ ${ }^{1}$ National Institute of Diabetes and Digestive and Kidney Diseases, National Institutes of Health, Bethesda, Maryland 20892, and ${ }^{2}$ Departments of \\ Psychiatry, Anesthesiology, Neuroscience, and Biomedical Engineering, Washington University School of Medicine, St. Louis, Missouri 63108
}

\begin{abstract}
The striatum is critical for controlling motor output. However, it remains unclear how striatal output neurons encode and facilitate movement. A prominent theory suggests that striatal units encode movements in bursts of activity near specific events, such as the start or end of actions. These bursts are theorized to gate or permit specific motor actions, thereby encoding and facilitating complex sequences of actions. An alternative theory has suggested that striatal neurons encode continuous changes in sensory or motor information with graded changes in firing rate. Supporting this theory, many striatal neurons exhibit such graded changes without bursting near specific actions. Here, we evaluated these two theories in the same recordings of mice (both male and female). We recorded single-unit and multiunit activity from the dorsomedial striatum of mice as they spontaneously explored an arena. We observed both types of encoding, although continuous encoding was more prevalent than bursting near movement initiation or termination. The majority of recorded units did not exhibit positive linear relationships with speed but instead exhibited nonlinear relationships that peaked at a range of locomotor speeds. Bulk calcium recordings of identified direct and indirect pathway neurons revealed similar speed tuning profiles, indicating that the heterogeneity in response profiles was not due to this genetic distinction. We conclude that continuous encoding of speed is a central component of movement encoding in the striatum.
\end{abstract}

Key words: electrophysiology; encoding; movement; speed; striatum; tuning

\section{Significance Statement}

The striatum is a structure that is linked to volitional movements and is a primary site of pathology in movement disorders. It remains unclear how striatal neurons encode motor parameters and use them to facilitate movement. Here, we evaluated two models for this: a "discrete encoding model" in which striatal neurons facilitate movements with brief burst of activity near the start and end of movements, and a "continuous encoding model," in which striatal neurons encode the sensory or motor state of the animal with continuous changes in firing. We found evidence primarily in support of the continuous encoding model. This may have implications for understanding the striatal control of movement, as well as informing therapeutic approaches for treating movement disorders.

\section{Introduction}

The striatum is implicated in controlling motor output and the speed or vigor of movements (Kravitz et al., 2010; Isomura et al.,

\footnotetext{
Received June 17, 2019; revised Dec. 20, 2019; accepted Jan. 2, 2020.

Author contributions:W.C.F., S.B., and A.V.K. designed research; W.C.F., S.B., J.A.L., N.L.M., B.A.M.-A., and M.C.C. performed research; W.C.F. and A.V.K. analyzed data; W.C.F. and A.V.K. wrote the first draft of the paper; W.C.F., S.B., J.A.L., N.L.M., B.A.M.-A., M.C.C., and A.V.K. edited the paper; W.C.F. and A.V.K. wrote the paper.

This work was supported by National Institutes of Health Intramural Research Program (National Institute of Diabetes and Digestive and Kidney Diseases). W.C.F. was supported by National Institutes of Health Postdoctoral Fellowship Center for Compulsive Behavior Postdoctoral Fellowship. S.B. was supported by Swiss National Science Foundation Early Postdoc.mobility fellowship P2GEP3_174898. This work was also supported by National Alliance for Research on Schizophrenia and Depression Young Investigator Grants to A.V.K. and M.C.C., Whitehall Foundation Grant 2017-12-54, Rita Allen Scholar Award, and R21 DA047127-01 to M.C.C. We thank the GENIE project at Janelia Research Campus for generous development and sharing of GCaMP reagents; and the National Institutes of Health Section on Instrumentation for designing and building the head-fixed wheel apparatus.
}

2013; Barbera et al., 2016; Lemos et al., 2016; Yttri and Dudman, 2016; Sales-Carbonell et al., 2018). Although multiple correlates of motion have been reported in striatal spike trains, it remains unclear how the striatum selects actions and/or controls the speed of actions. Two theoretical models have emerged. According to one, ensembles of striatal neurons encode and promote specific components of movement, such that distinct populations of neurons fire bursts of activity at discrete points in motor sequences, for example, the initiation or termination of the sequence (Jog et al., 1999; Schultz et al., 2003; Costa et al., 2004;

The authors declare no competing financial interests.

Correspondence should be addressed to Alexxai V. Kravitz at alexxai@email.wustl.edu.

https://doi.org/10.1523/JNEUROSCI.1407-19.2020

Copyright $\odot 2020$ the authors 
Barnes et al., 2005; Jin and Costa, 2010; Cui et al., 2013; Geddes et al., 2018; Martiros et al., 2018). These bursts of activity are thought to gate or permit specific actions and thereby facilitate chains of motor sequences, possibly through disinhibition of downstream structures (Mink, 1996).

Although consistent with recordings performed during goaldirected tasks, deficiencies in this model have been noted (Robbe, 2018; Sales-Carbonell et al., 2018). First, striatal neurons respond to stimuli unrelated to purposive actions, including passive movements of limbs (Carelli and West, 1991; Coffey et al., 2016; Kulik et al., 2017). Second, many striatal neurons contain information about continuous movement variables, such as speed or acceleration, without bursting at transition points (Kim et al., 2014; Panigrahi et al., 2015; Rueda-Orozco and Robbe, 2015; Barbera et al., 2016; Klaus et al., 2017; Markowitz et al., 2018; SalesCarbonell et al., 2018). Finally, burst firing is often weak or undetectable early in task learning when behavioral stereotypy is low, even though animals perform similar actions during these phases (Barnes et al., 2005; Jin and Costa, 2010). A second model proposes that striatal neurons represent continuous motor variables and can appear to burst near specific actions due to rapid changes in these variables around those actions (Rueda-Orozco and Robbe, 2015; Markowitz et al., 2018; Sales-Carbonell et al., 2018). This "continuous encoding model" suggests that ensembles select actions and govern their speeds in an ongoing fashion, through graded changes in firing. Continuous encoding of speed has also been described in the midbrain (Puryear et al., 2010) and cortex (Moran and Schwartz, 1999), which both project to the striatum.

Here, we evaluated these two models by determining the relative contributions of discrete and continuous encoding of locomotion in the dorsomedial striatum (DMS) of mice. We recorded single and multiunits from the DMS of freely moving mice as they spontaneously explored an arena. We observed both types of encoding, but a larger percentage of striatal neurons participated in a continuous representation than in discrete signaling near the start or end of locomotion. Although a large literature links striatal firing to increased locomotion (Costa et al., 2004; Shi et al., 2004; Yamin et al., 2013; Kim et al., 2014; Rueda-Orozco and Robbe, 2015; Barbera et al., 2016; Parker et al., 2018), most DMS units did not exhibit positive linear relationships between firing and locomotor speed but instead exhibited nonlinear relationships that peaked across a range of speeds. Based on opposing actions of the two populations of output projection neurons (Kravitz et al., 2010; Yttri and Dudman, 2016), we tested whether direct and indirect pathway medium spiny neurons (dMSNs and iMSNs) might be tuned to different locomotor speeds. To quantify this, we recorded population calcium activity from dMSNs and iMSNs and found that the variability in speed tuning was not explained by these populations. Finally, we assessed whether nonlinear speed tuning was also present during head-fixed constrained locomotion, and observed speed tuning curves during this condition as well. Our results support the "continuous encoding model" (Robbe, 2018), whereby DMS neurons represent information about the ongoing sensory and motor state of the animal with continuous changes in firing rate.

\section{Materials and Methods}

\section{Experimental model and subject details}

Male and female mice (C57BL/6 background, p40-p180) were maintained under standard conditions $\left(12 \mathrm{~h}\right.$ light/dark cycle, $\left.23^{\circ} \mathrm{C}-25^{\circ} \mathrm{C}\right)$ with mouse chow and water provided ad libitum. WT mice were used for the in vivo electrophysiology experiments $(n=13)$. A2A-cre $(n=5)$,
D1-cre $(n=6)$, and WT $(n=3)$ mice were used in the photometry experiment (Gong et al., 2007). Animal care and procedures were approved by the Animal Care and Use Committee of National Institute on Diabetes and Digestive and Kidney Diseases and Washington University in St Louis.

\section{Method details}

Array and fiber implantations. Mice were anesthetized with isoflurane $(2 \%-3 \% \mathrm{v} / \mathrm{v}$ for induction and $1 \%$ for maintenance $)$ and placed in a stereotaxic frame (Stoelting). Once anesthetized, the skull was exposed by making a small incision, and holes were drilled for electrode implantation. An array with 32 Teflon-coated tungsten microwires $(35 \mu \mathrm{m}$ diameter; Innovative Neurophysiology) was implanted into the right DMS (AP, $0.5 \mathrm{~mm}$; ML, $1.5 \mathrm{~mm}$; DV , $-2.6 \mathrm{~mm}$ ). For photometry mice, $500 \mathrm{nl}$ of Cre-dependent GCaMP6s (AAVDJ-Efla-DIO-GCaMPs, $3.1 \times$ $10^{12} \mathrm{vg} / \mathrm{ml}$ ) was infused and optic fiber cannulas (fiber: $200 \mu \mathrm{m}$ core, $0.48 \mathrm{NA}$; Doric Lenses) were implanted in the right DMS (AP, $0.5 \mathrm{~mm}$; $\mathrm{ML}, 1.5 \mathrm{~mm}$; DV , $-2.6 \mathrm{~mm}$ ). Virus was purchased from the UNC Vector Core. After infusion, injector tips were left in place for an additional $5 \mathrm{~min}$ to encourage spread before injector withdrawal. Optic fibers and electrode arrays were secured to skull with a thin layer of adhesive dental cement (C\&B Metabond, Parkell) followed by a layer of cranioplastic cement (Stoelting). Stainless-steel head-bars for mice in Figure 6 were attached to the canioplastic cement with UV-curable epoxy. Following surgeries, mice were returned to home cages for at least 1 week for recovery before any experiments progressed.

In vivo electrophysiology. Neurophysiological signals were transmitted to an Omniplex neurophysiology system (Plexon) via a multiplexing headstage (Triangle Biosystems M32 for the experiments in Figs. 1, 2, 3, 4, 5; Plexon digital headstage for the head-fixed experiments in Fig. 6). Spike channels were acquired at $40 \mathrm{kHz}$ with 16 -bit resolution, and bandpass filtered at $150 \mathrm{~Hz}$ to $3 \mathrm{kHz}$ before spike sorting. Local field potential signals were simultaneously digitized at $1 \mathrm{kHz}$. Single and multiple units were discriminated using principal component analysis (Offline Sorter; Plexon), using MANCOVA to determine whether single-unit clusters were statistically distinct from multiunit clusters. Single units were further classified into MSNs and interneurons based on spike waveform shape, in that, if the trough-peak duration was $<350 \mu$ s, the unit was considered a putative interneuron, and otherwise a putative MSN. Mice were recorded four times, and the recording with the largest number of channels with unit activity was chosen for analysis.

Fiber photometry. A single optic fiber $(200 \mu \mathrm{m}$ core, $0.48 \mathrm{NA}$, Doric Lenses) was used to transmit excitation and emission light. Mice were plugged in with a zirconia mating sleeve. Blue light was generated by a light-emitting diode (Plexon Plexbright $465 \mathrm{~nm}, 15-30 \mu \mathrm{W}$ continuous light). A dichroic mirror and 505-535 nm filter (FMC4 port minicube, Doric Lenses) were used to parse emitted green fluorescent light, which was measured with a photodetector (model 2151, Newport). Output signals were captured, amplified, and recorded with a digital acquisition system (Omniplex, Plexon). Fluorescence data were normalized and converted to both $Z$ scores and normalized change in fluorescence $(\mathrm{df} / \mathrm{F})$ using a custom scripts run in NeuroExplorer.

\section{Open field and head-fixed behavior}

For open-field recordings, mice were plugged in and allowed to freely explore a $30 \mathrm{~cm} \times 30 \mathrm{~cm}$ open-field arena. Mice were all plugged in for at least 3 times before the recording session, to habituate them to the weight of the headstage and recording cable. Mice were videotaped overhead with a camera recording video at $30 \mathrm{~Hz}$, videos were processed in Noldus Ethovision, and speed data were synchronized and aligned with the electrophysiology data. For head-fixed recordings, mice were gradually habituated to being head-fixed over a period of 2-3 weeks. This started with brief (1-2 min) sessions of head fixation, expanding to 30-60 min with daily sessions over 2-3 weeks. The head fixation wheel was a custom wheel ( 8 inches in diameter) mounted in an aluminum frame with bilateral steel head fixation bars (National Institutes of Health Section on Instrumentation). It was equipped with an infrared sensor mechanism that exported a digital TTL pulse every 5 degrees of wheel rotation $(0.6$ $\mathrm{cm}$ of wheel distance). These TTL pulses were captured by the Omniplex 
electrophysiology system in real-time and processed offline to generate a smoothed curve of wheel speed across the session. On the recording day, mice were head-fixed and allowed to freely locomote for 30-60 min, after which they were removed from the head fixation wheel and immediately placed in an open field for an additional 60 min recording.

\section{In vivo electrophysiology data analysis}

Locomotion bout start and end responses. Start and end of locomotion bouts were identified in a two-stage process. First, peaks in the speed signal that broke $5 \mathrm{~cm} / \mathrm{s}$ were identified to verify that the bout contained locomotion. Next, we scanned backwards in time from this peak in $0.1 \mathrm{~s}$ bins to identify the first time point $>2 \mathrm{~cm} / \mathrm{s}$, which was used as the start of the locomotion bout, and scanned forward in time to identify the last time point $>2 \mathrm{~cm} / \mathrm{s}$, which was considered the end of the locomotion bout (for graphical representations, see Fig. 2A). To identify neurons that increased firing near the start or end of locomotion, we exported the average peristimulus time histogram of spiking of each unit around each event from -10 to $30 \mathrm{~s}$, in $0.1 \mathrm{~s}$ bins. We defined the period from -5 to $-2 \mathrm{~s}$ as "baseline" and -1 to $2 \mathrm{~s}$ as "signal." We extracted the distribution of firing rates in baseline period and scanned a moving window of $0.1 \mathrm{~s}$ across the signal period, identifying units that had at least 3 consecutive bins (i.e., $300 \mathrm{~ms}$ in a row) above the 95th percentile of the baseline distribution. Permutation analyses were run identically, with the peristimulus time histogram data shuffled before each permutation. Python analysis code and data are available at https://osf.io/dfxkp/.

Speed tuning of spiking units. Speed and spike rates for each unit were binned into $0.1 \mathrm{~s}$ bins in Neuroexplorer and saved as CSV files for import into Python 3.7. In Python 3.7, speeds were classified into 50 equally spaced bins ranging from 5 to $15 \mathrm{~cm} / \mathrm{s}$, with a $0.2 \mathrm{~cm} / \mathrm{s}$ bin width, using the pandas.cut funtion. Data at speeds $<5 \mathrm{~cm} / \mathrm{s}$ were excluded from this analysis. The firing rate within each speed bin was calculated for each unit, and scatterplots were generated between speed and neural firing. Both linear and quadratic regressions were performed on the resulting data using numpy.polyfit, and regression statistics were saved for later analysis. Significant fits were determined based on $p<0.01$. Thirteen units were excluded from analyses due to firing rates $<0.001 \mathrm{~Hz}$, as these were overfit by the quadratic regression. Permutation analyses were run 1000 times with speed data shuffled before each permutation.

Speed predictions from spiking data. Both speed and spiking data were binned into $0.1 \mathrm{~s}$ bins in Neuroexplorer and saved as CSV files for import into Python 3.7. In Python 3.7, the data were rebinned into $10 \mathrm{~s}$ bins and split into "training" and "testing" sets of 30 min each (additional analyses were completed with different bin widths and training set sizes, which are presented in Fig. 5D,E). For average "per-mouse" spiking and photometry predictions, quadratic regressions were run between speed and neural activity in the training set, and the resulting quadratic coefficients were used to calculate the predicted speed from neural activity in the testing set. For multiunit population predictions, firing rates of all single and multiunits in the training data were regressed on velocity with a multilinear regression (from the Python sklearn library). The resulting regression coefficients were used to calculate predicted speed in the testing data from firing rates of these same units. One constraint was applied to the predicted speeds in all cases, in that negative predictions for speed were set to $0 \mathrm{~cm} / \mathrm{s}$. The performance of the predictive modeling was evaluated with linear regressions between predicted speeds and actual speeds in the testing data to generate $R^{2}$ values and root mean square error.

Histology. Following all experiments, mice were killed. Mice with electrode arrays received electrolytic lesions $(100 \mu \mathrm{A}$ for $5 \mathrm{~s}$, Ugo Basile Lesion Making Device) to assist in identification of array placements. Brains were removed and fixed in $10 \%$ formalin for $16-24 \mathrm{~h}$. Brains were transferred to $30 \%$ sucrose in PBS for $2-3 \mathrm{~d}$ and sectioned at $40 \mu \mathrm{m}$ on a freezing microtome (Leica Microsystems). Sections were counterstained with DAPI and mounted on slides for imaging with a slide-scanning microscope (Olympus VS120) at $10 \times$. Fluorescence areas from slide scans were outlined in ImageJ (https://imagej.nih.gov/ij/) and positioned over a corresponding atlas section in Illustrator (Adobe). Microwire array positions were identified by damage to the striatum around the lesion.

\section{Quantification and statistical analysis}

Statistical analysis was performed using Python 3.7 or GraphPad Prism (version 8.1, GraphPad Software). Fisher's exact test, twotailed paired $t$ tests, one-way repeated-measures ANOVAs, or two-way repeated-measures ANOVAs were used when appropriate and as stated in the Results and figure legends. ANOVAs were performed with Tukey's multiple post hoc comparisons. Results were considered significant at an $\alpha$ of $p<0.05$.

Data and software availability

Python analysis code and data are available at https://osf.io/dfxkp/.

\section{Results}

\section{Striatal firing reflects both discrete and continuous changes} in locomotion

To understand the behavior of mice while exploring the open field, we video recorded $4 \mathrm{WT}$ mice (C57BL6 background) for $1 \mathrm{~h}$ in a $30 \mathrm{~cm}$ square open-field and used a machine learning-based behavioral classifier (Noldus Information Technologies) to classify behavior into five categories: digging, grooming, rearing, resting, and walking (Fig. $1 A-C$ ). We compared the speed distribution of each behavior, determining that speeds $<5 \mathrm{~cm} / \mathrm{s}$ contain a mix of all five behaviors, whereas speeds $>5 \mathrm{~cm} / \mathrm{s}$ are $>92 \%$ locomotion (Fig. 1C). We defined all data at speed $>5 \mathrm{~cm} / \mathrm{s}$ as locomotion for further analyses. We recorded neural activity from 9 additional mice who were implanted with 32 electrode microwire arrays in the dorsal medial striatum (Fig. 1D) and recorded while they explored the open field for $1 \mathrm{~h}$. A total of 50 single units and 168 multiunits were recorded ( 1 recording per mouse; Fig. $1 E$ ). Based on waveform shape (Berke et al., 2004), single units were classified into 7 putative interneurons and 43 putative MSNs (Fig. 1F; see Materials and Methods).

We asked two questions regarding the relationships of spiking activity and locomotion: (1) Do striatal units exhibit discrete changes in firing near the start and end of locomotion in the open field, as observed previously in operant tasks? (2) Does firing of striatal units correlate with speed, independently of discrete start or end responses? To evaluate the first question, we identified bouts of locomotion $(>5 \mathrm{~cm} / \mathrm{s})$, as well as the start and end of each bout (defined as when the speed broke $2 \mathrm{~cm} / \mathrm{s}$; Fig. $2 A$ ). We tested each recorded single and multiunit for a phasic increase in firing near this event $(n=211)$, using a procedure based on that used by Jin and Costa (2010). Thirty-eight of 211 (18\%) recorded units increased firing near the start of locomotion (Fig. $2 \mathrm{~B}, \mathrm{C}$ ). To evaluate the stringency of our detection criteria, we performed a permutation analysis in which we shuffled the speed data 1000 times, detecting an average of $2.9 \%$ responsive neurons. In similar analyses near locomotion end, 31 of 211 (15\%) units increased firing near this event (Fig. $2 E, F$ ). Again, a permutation analysis detected $2.4 \%$ of units as responsive. From these analyses, we conclude that: (1) striatal units exhibit discrete increases in firing near the start and end of locomotion in the open field; and (2) changes in firing of these neurons closely resemble the rapid changes in speed near the start and end of locomotion (Fig. $2 C)$. This supports the possibility that discrete changes in firing may reflect continuous representations of motor parameters such as speed, a view that has been previously advanced (RuedaOrozco and Robbe, 2015; Sales-Carbonell et al., 2018).

To evaluate this possibility, we quantified relationships between striatal firing rates and locomotor speed in the open field in a continuous manner. Based on literature reports of linear relationships between striatal activity and movement speed (Costa et al., 2004; Shi et al., 2004; Kim et al., 2014; Panigrahi et al., 2015; Rueda-Orozco and Robbe, 2015; Bartholomew et al., 2016; 
A

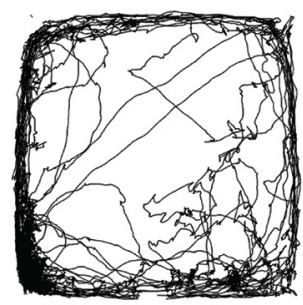

1 hour of open field

D

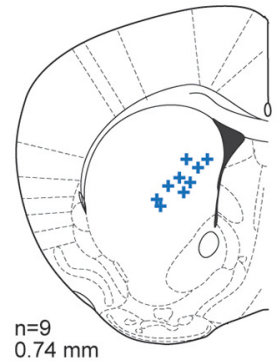

B

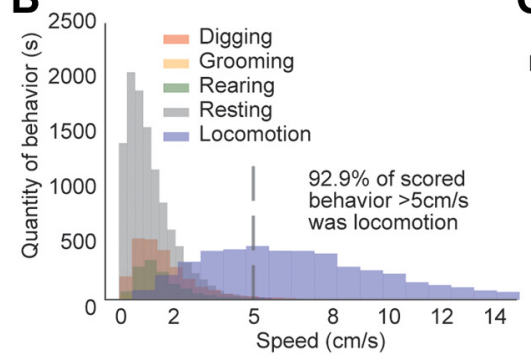

C

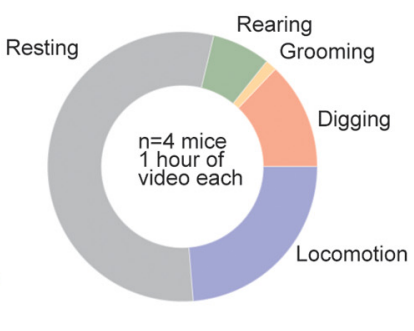

$E$

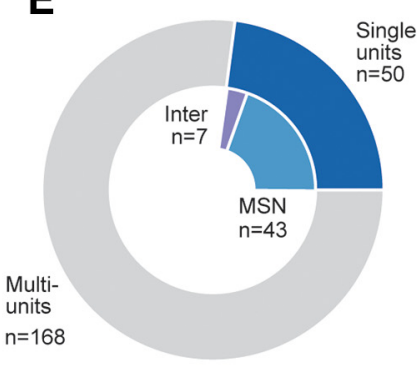

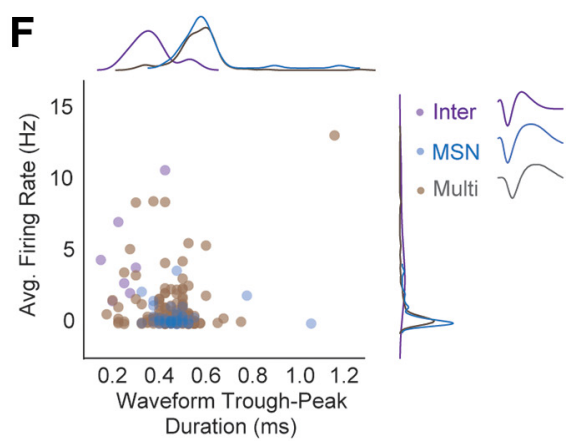

Figure 1. In vivo recordings of neural activity during free behavior in the open field. $\boldsymbol{A}$, Example track plot of open-field behavior. $\boldsymbol{B}$, Histogram analysis quantifying amount of distinct behaviors occurring at different speeds. C, Proportion of $1 \mathrm{~h}$ open-field time spent engaging in different behaviors. $\boldsymbol{D}$, Electrode array placements. + , Histological estimate of center of array. Image adapted from Franklin and Paxinos (2019). $\boldsymbol{E}$, Number of recorded striatal units, including multiunits, putative MSNs, and putative interneurons. $\boldsymbol{F}$, Classification of single units into putative MSNs and interneurons by waveform shape and firing rate.

\section{A}

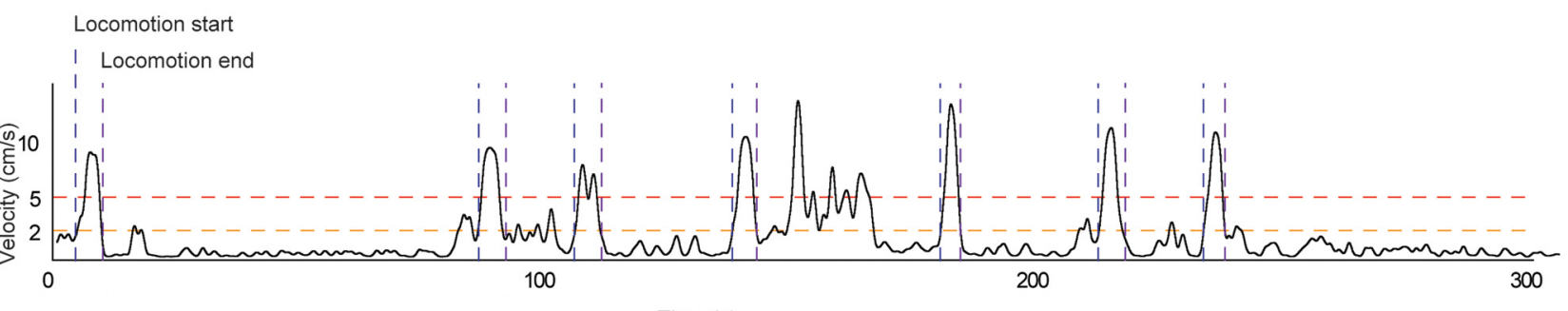

B

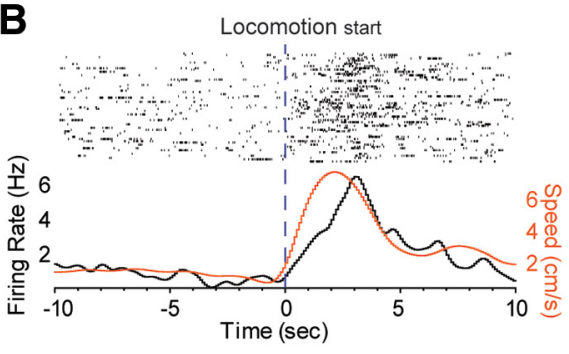

E

Locomotion end

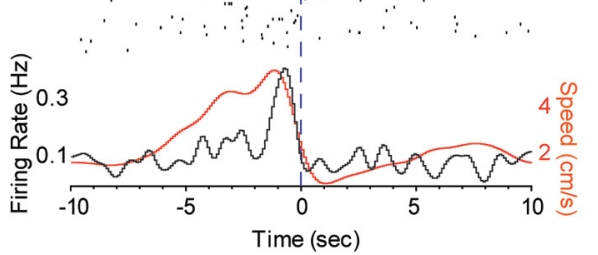

Time (s)

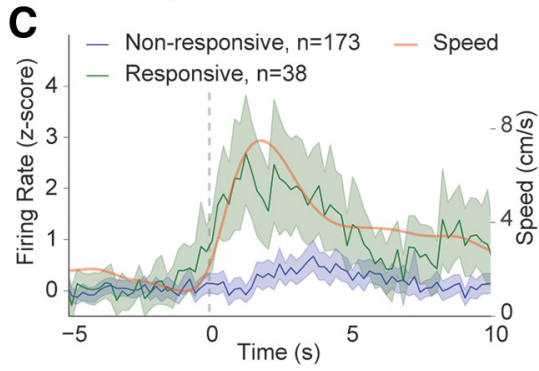

F $\quad$ - Non-responsive, $n=180 \quad$ - Speed

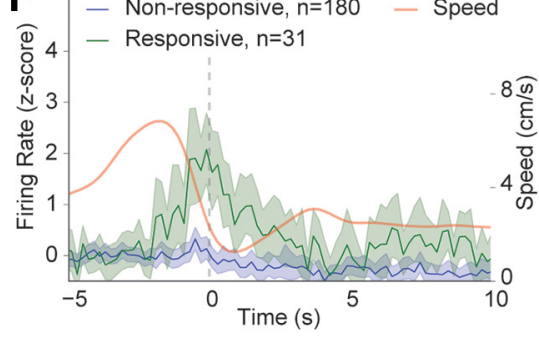

D

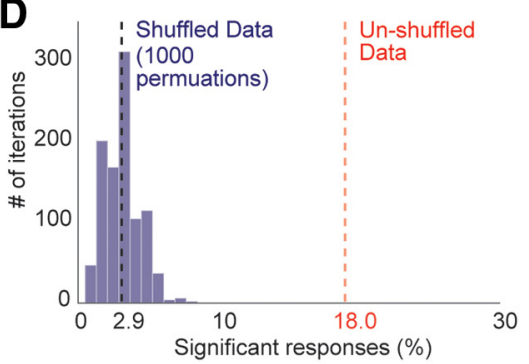

G

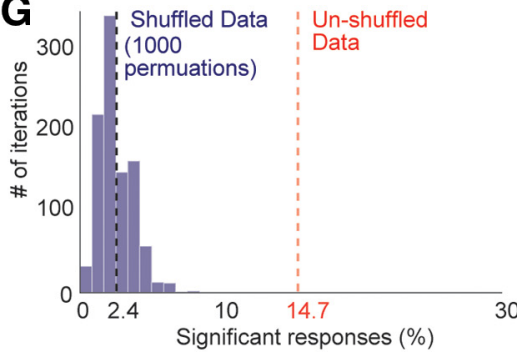

Figure 2. Striatal units exhibit phasic responses near starts and ends of locomotion. $A$, Example speed trace with lines indicating periods of locomotor behavior and start and end criteria (see Materials and Methods). $\boldsymbol{B}$, Example striatal unit firing and speed near locomotion start. $\boldsymbol{C}$, Average firing rate and speed for striatal units which do and do not exhibit significant modulation near locomotion start. Shaded regions represent $\pm 95 \%$ Cl. D. Permutation analysis showing the number of units with significant phasic responses near locomotion start after shuffling speed data $1000 \times$. Black dashed line indicates the shuffled mean. Red dashed line indicates the unshuffled number (Fisher's exact test for locomotion starts, shuffled average of 6.1 of $211 \mathrm{vs} 38$ of $211, p<$ 0.0001 , locomotion ends, 5.1 of 211 vs 31 of $211, p<0.0001)$. $\boldsymbol{E}-\mathbf{G}$, Same analysis and graphical representation as in $\boldsymbol{B}-\boldsymbol{D}$, but for locomotion end. 
A Examples of simultaneously recorded units with continuous coding of speed
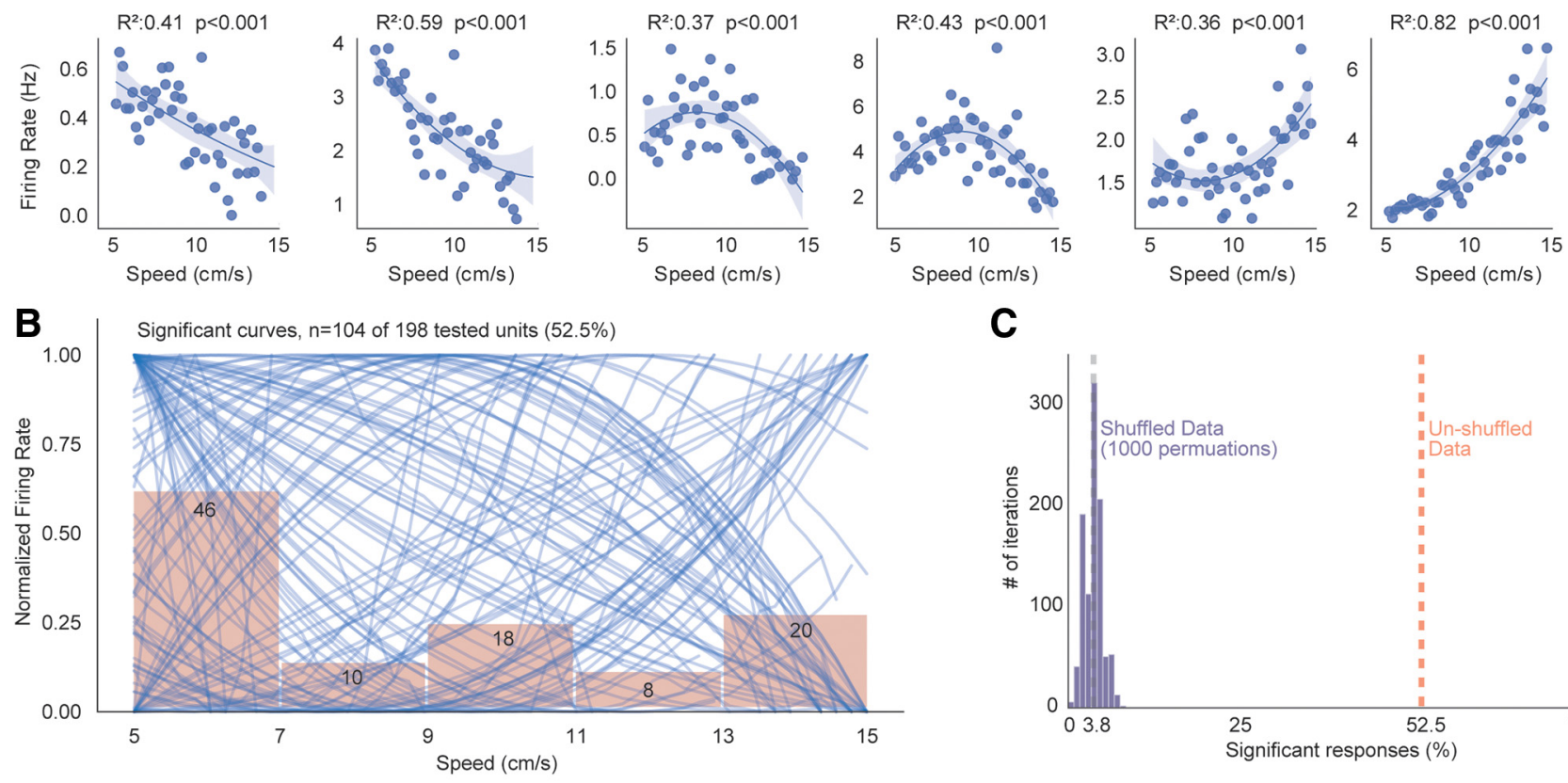

C

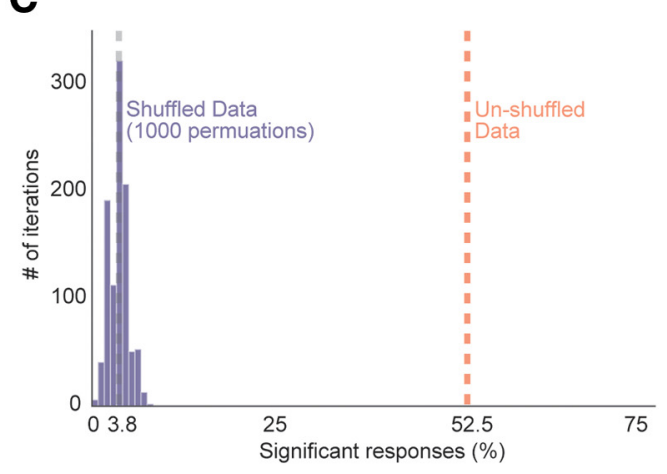

D

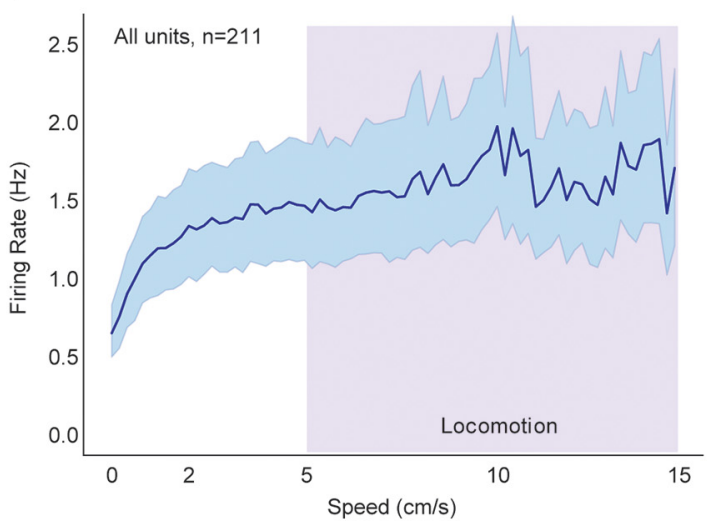

E

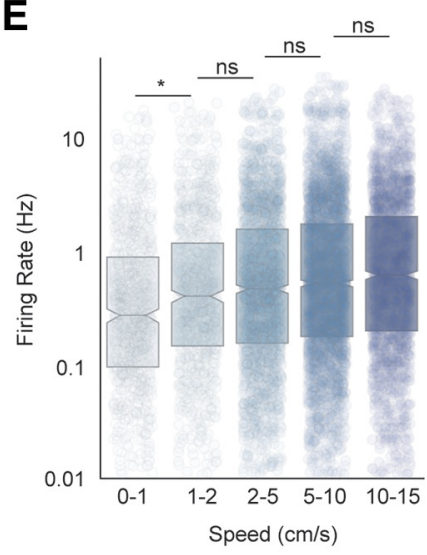

F

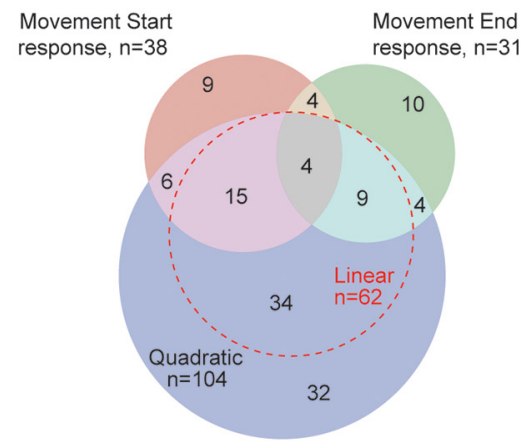

Figure 3. Striatal firing correlates with locomotion speed. $\boldsymbol{A}$, Example striatal units with significant quadratic relationships between firing rate and speed (speed tuning curves). Shaded regions represent $\pm 95 \%$ Cl. $\boldsymbol{B}$, Significant speed tuning curves $(n=104)$ for MSNs overlaid with bar graphs reflecting the number of units whose tuning curves peak in given speed range. $\boldsymbol{C}$, Permutation analysis showing the number of striatal units with significant nonlinear regressions after shuffling speed data. Black dashed line indicates the shuffled mean. Red dashed line indicates the unshuffled number (Fisher's exact test, 7.52 of 198 vs 104 of $198, p<0.0001$ ). D, Average firing rates of all striatal units from 0 to $15 \mathrm{~cm} / \mathrm{s}$. Data are mean $\pm 95 \% \mathrm{Cl}$. Shaded region represents locomotion speed range. $\boldsymbol{E}$, Average firing rates of striatal units during five different locomotion speed ranges. One-way repeated mixed-model AN0VA, main effect of speed $\left(n=211, F_{(4,773)}=11.94, p<0.0001\right)$. $\boldsymbol{F}$, Venn diagram showing number of striatal units with significant phasic responses near start and ends of locomotion bouts and number of striatal units with significant nonlinear speed correlations. Dashed red line indicates number of striatal units with significant linear correlations. Fisher's exact test, 104 of 211 speed correlated versus 61 of 211 start/end-modulated, $p<0.001$. Box plots: box represents $25-75$ percentiles; line indicates median. ${ }^{*} p<0.05$ (significant post hoc comparison). ns, Not significant at $p>0.5$.

Sales-Carbonell et al., 2018), we first modeled these relationships with linear correlations. Sixty-two (29\%) of the recorded units exhibited a significant linear correlation with movement speed, with a slightly larger number of units firing more to low speeds of locomotion (negative slope) than to high speeds (Fisher's exact test for direction of linear relationship, 38 of 62 negative vs 24 of 62 positive, $p=0.07$; Fig. $3 F$ ). Therefore, the relationship between striatal spiking and speed was not monotonically positive, although this distinction was not quite significant.

\section{Individual striatal units are tuned to a range of locomotor speeds}

We also observed many neurons with nonlinear relationships that fired most strongly to intermediate locomotor speeds (Fig. $3 A, B)$. We tested whether striatal units would exhibit "speed tuning," similar to patterns detected in brain areas that project to the striatum (Moran and Schwartz, 1999; Puryear et al., 2010). To test this, we fit these relationships with quadratic regressions, which revealed 104 (52.5\%) units with significant quadratic relationships between spiking and speed (Fig. 3B). The peak firing of individual units tiled the entire range of speeds, with the largest proportion peaking at low-speed locomotion $(5-7 \mathrm{~cm} / \mathrm{s} ;$ Fig. $3 B)$. We validated this quadratic regression approach with a permutation analysis in which we shuffled the speed data and recalculated the curves 1000 times. This captured a significantly lower number of quadratic relationships (mean from shuffled data $=$ 3.8\%; Fig. 3C). These analyses argue against a monotonically increasing firing rate with increased speed of locomotion and highlight the range of locomotor speeds to which individual units are tuned. To evaluate this property with an orthogonal analysis, we compared average firing rates of all recorded units across the entire range of open field speeds $(0-15 \mathrm{~cm} / \mathrm{s}$; Fig. 3D). By 
repeated-measures ANOVA, there was a significant effect of speed across all units $\left(n=211, F_{(4,773)}=11.94, p<0.0001\right)$. In post hoc testing (Tukey's multiple-comparison test), there was a significant increase in spiking between $0-1$ and $1-2$ $\mathrm{cm} / \mathrm{s}(p=0.01)$, but not between 1-2 and $2-5 \mathrm{~cm} / \mathrm{s}, 2-5$ and $5-10 \mathrm{~cm} / \mathrm{s}$, and $5-10$ and $10-15 \mathrm{~cm} / \mathrm{s}$ (all $p>0.48$; Fig. $3 D, E$ ).

Finally, we counted the number of recorded units that exhibited each type of encoding. A large proportion of MSNs exhibited at least one form of locomotion encoding (127 units, 60\%), with more neurons participating in continuous (quadratic) than discrete (Movement Start/ Movement End responses) encoding (Fisher's exact test, 104 of 211 vs 61 of 211 , $p<0.0001$; Fig. $3 F)$. In addition, a large group of neurons $(n=66)$ exhibited correlations with speed without any detectable increase near the locomotion start or end (Fig. 3F). Finally, more than half of the neurons that exhibited discrete signaling near the locomotion start or end also exhibited correlations with speed (38 of $61)$. Thus, our data suggest that striatal activity contains a continuous representation of speed, and that some neurons that appear to exhibit phasic responses to locomotion start or end may indeed be reflecting this continuous representation.

\section{Population calcium activity of dMSNs and iMSNs similarly encode speed}

Based on the opposing actions of dMSNs and iMSNs in governing motor output (Kravitz et al., 2010; Meng et al., 2018; Bariselli et al., 2019), we asked whether the diversity in speed tuning might be explained by opposing activity in these two populations. Specifically, we asked whether dMSNs would be tuned to higherspeed locomotion and iMSNs tuned to rest or lower-speed locomotion. To evaluate this prediction, we used fiber photometry to record bulk calcium activity from each population as mice explored an open field (Fig. $4 A-C$ ). The average relationship between activity and speed varied positively in both populations (Fig. 4D), with both resembling that of the total spiking population (Fig. 3D,E). By two-way repeated-measures ANOVA, we detected a significant effect of speed across the two GCaMP6sexpressing groups $\left(F_{(1.177,10.60)}=6.771, p=0.022\right)$, but no significant effect of genotype or genotype $\times$ speed interaction (both $p>0.44$ ). With post hoc tests (Tukey's multiple-comparisons tests), the increase in speed was driven by an increase in fluorescence between $0-1 \mathrm{~cm} / \mathrm{s}$ and $1-2 \mathrm{~cm} / \mathrm{s}$ in both groups $(p<0.05$ for both), without further increases in fluorescence across the other speeds ( $p>0.55$ for all). In conclusion, the diversity in speed tuning is not explained by differential tuning in dMSNs versus iMSNs, at least at the population level.

\section{Population codes, not average activity, predict speed}

As the average striatal population activity did not differentiate between different speeds of locomotion (Fig. 3D,E), we hypothesized that the speed tuning of individual units may contain more reliable information on speed. To evaluate this, we performed a speed decoding analysis (see Materials and Methods). Here, we calculated the coefficients of quadratic regression between spik-
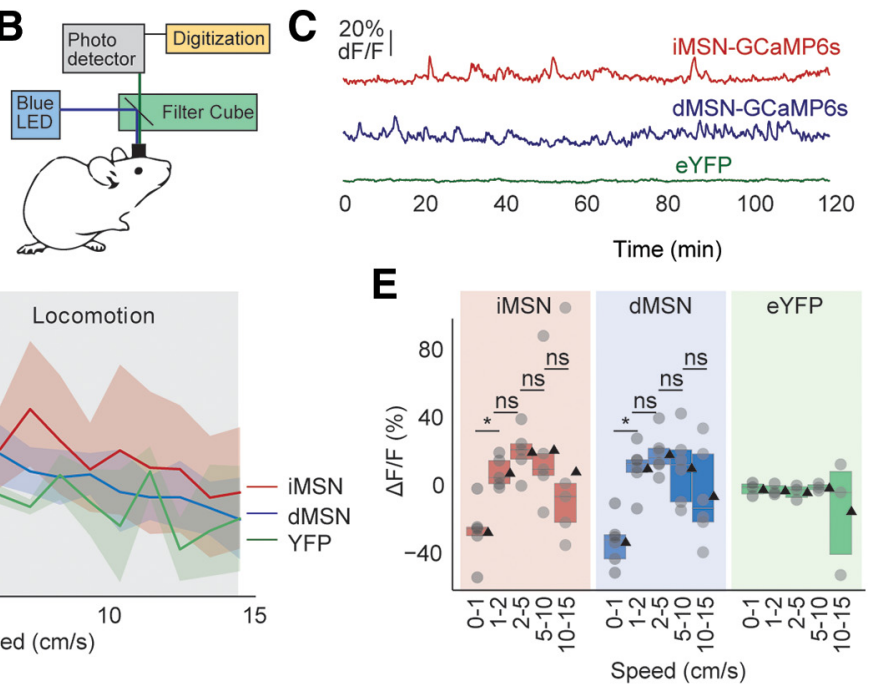

Figure 4. Direct and indirect pathway activity has similar relationships with locomotion speed. $\boldsymbol{A}$, Example histology of t locomotion speed ranges. Box plots: box represents $25-75$ percentiles; line indicates median; black triangles represent means. ${ }^{*} p<0.05$ (significant post hoc comparison). ns, Not significant at $p>0.5$.

ing and speed in the first 30 min of data (training set) and tested how accurately those coefficients predicted the animal's speed in the second $30 \mathrm{~min}$ of data (testing set). We first performed this analysis on the average of all unit activity for each session which weakly predicted speed (average $R^{2}=0.25, r=0.41$; Fig. $5 A-C$ ). Next, to evaluate our hypothesis that a population code would better predict speed than the simple population average in each session, we performed a multilinear regression that included the spiking activity of all units as regressors, and speed as the dependent variable. This model performed significantly better than the average population firing rate (average $R^{2}=0.52, r=0.70$, both $p<0.01$ vs predictions from average $\mathrm{Hz}$; Fig. $5 A-C$ ). Two examples comparing this model to the prediction obtained from average spiking are presented in Figure $5 C$.

To better understand variables that might influence speed predictions, we varied (1) the bin size and (2) the size of the held-out testing set. Larger bin sizes performed better, suggesting that striatal population codes are better at predicting longer-lasting states of locomotion than second-by-second changes in speed (Fig. $5 D$ ). Not surprisingly, the predictive model also performed better with larger periods of training data, although it performed surprisingly well when trained on as little as $10 \mathrm{~min}$ of data (Fig. $5 E$ ). Finally, we ran the quadratic regression prediction on the population calcium fluorescence of dMSNs and iMSNs. The predictive ability of these traces was also weak (all average $R^{2}<0.10$; Fig. $5 F$ ), similar to that of average spiking. We conclude that population codes are more reliable for decoding speed information than average firing rates in the striatum, or average activity of either population of projection neurons.

\section{Nonlinear speed tuning is also present during head- fixed locomotion}

To examine continuous coding in a more constrained behavioral context, we performed an additional set of recordings in animals that were first head-fixed on a wheel and then detached and allowed to explore an open field (Fig. 6A). Our primary question was whether a range of speed tuning is also present during head- 
A

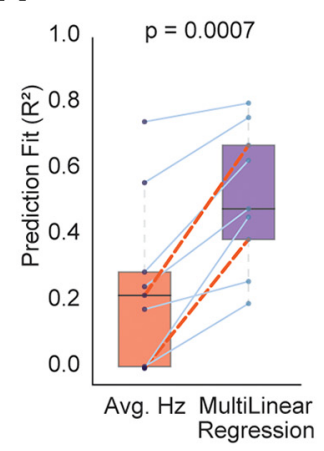

B

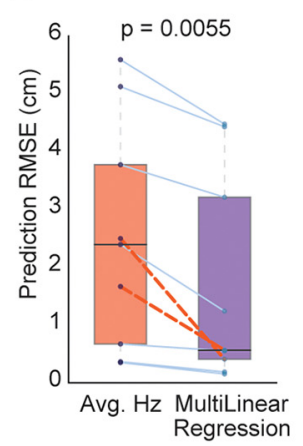

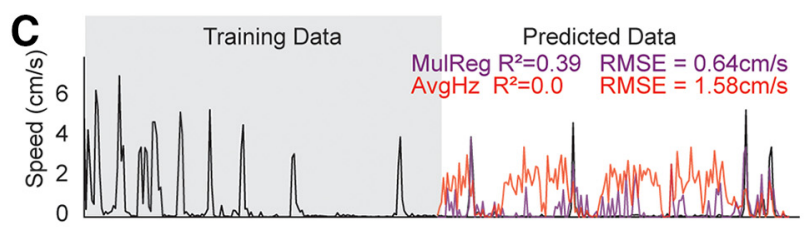

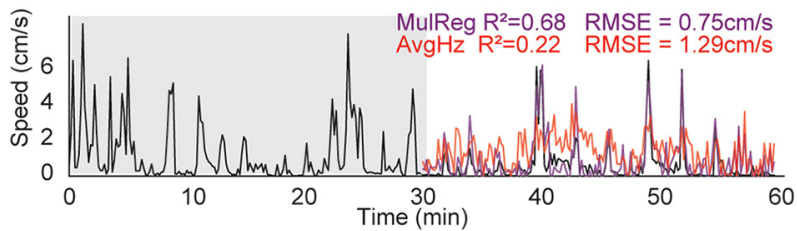

$\mathbf{D}_{0.7}$

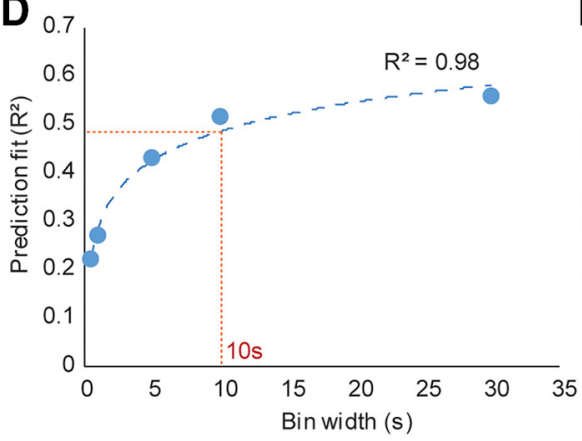

$E_{00}$

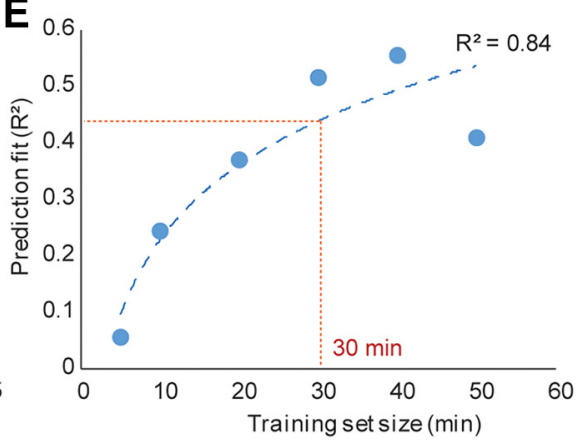

F Speed predictions

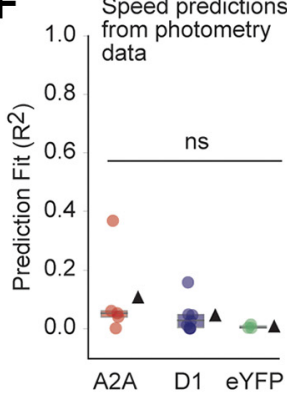

Figure 5. Population codes, not average activity, predict speed. A, Prediction fits $\left(R^{2}\right)$ for measured speeds using the average firing rate of all striatal units from each mouse or a multilinear regression of the same units per mouse. Paired two-tailed $t$ test, $p<0.0007$. $B$, Root mean square error (RMSE) for predicted speeds using average firing rate or a multilinear regression of all units from each mouse. Paired two-tailed $t$ test, $p=0.0055$. C, Example speed predictions from 2 mice, comparing speed predicted by the average firing rate versus the multilinear regression. Orange dashed lines in $\boldsymbol{A}$ and $\boldsymbol{B}$ indicate these examples in the larger dataset. $\boldsymbol{D}, \boldsymbol{E}$, Average multilinear regression prediction fits ( $n=9$ mice, 9 sessions) generated by varying the bin width of the training data $(\boldsymbol{D})$, or duration of training dataset $(\boldsymbol{E})$. D-E, Dashed blue lines indicate semilog fits. Dashed red lines indicate parameters used to generate fits. $\boldsymbol{F}$, Linear regression predictions of speed from photometry data, one-way ANOVA $\left(F_{(2,11)}=1.023, p=0.39\right)$. Box plots: box represents $25-75$ percentiles; line indicates median; black triangles represent means. ns, Not significant at $p>0.5$.

fixed locomotion. We recorded 34 single and multiunits from 4 mice in this experiment. As expected from prior work (Kim et al., 2014; Sales-Carbonell et al., 2018), we observed discrete changes in firing near the start and end of locomotion on the head-fixed wheel (Fig. 6B), with the average spiking closely resembling the speed profile of the wheel near both events. We performed a quadratic regression between striatal firing rate and wheel running speed and noted that 13 of 34 units (38\%) exhibited significant speed correlations that peaked at a range of speeds (Fig. $6 C$ ), similar to the diverse tuning curves observed previously in the open field (Fig. 3A). Next, we analyzed these same units in the open-field period of the recording, and identified 16 units (47\%) that exhibited speed tuning, with 7 (21\%) exhibiting speed tuning in both settings. As the majority of speed correlated units (15 of $22,68 \%$ ) exhibited speed correlations in one but not both locomotor contexts (Fig. 6D), our data lend additional support to the idea that striatal units likely do not represent speed in an absolute sense, but rather represent speed in a multiplexed, context-dependent manner that may include sensory and kinematic information relevant to the ongoing context (Turner and Desmurget, 2010; Rueda-Orozco and Robbe, 2015). Supporting the concept of context-dependent signaling in these units, average firing rates of striatal units were significantly higher in the open-field period relative to the head-fixed period (Fig. 6E,F).

\section{Discussion}

In the current study, we evaluated two hypotheses for striatal motor encoding in mice exploring an open field, a condition that does not produce highly stereotyped sequences of actions nor expose animals to predictable stimuli that can entrain neuronal firing. While there were DMS units that showed discrete changes in firing near the start and end of locomotion, most of the units exhibited graded changes in firing that correlated with locomotor speed. The relationship between the continuous firing patterns and speed were nonlinear and predominately tuned to low-speed walking; and the population activity, but not average multiunit firing, was predictive of locomotor speed. Additionally, when DMS units were analyzed in mice that were head-fixed to a wheel, which limited the sequences of actions they could undertake, before exploring an open field, similar speed tuning was observed. Thus, our results support a role for the DMS in representing ongoing motor and sensory information in a continuous manner and highlight limitations in some previous approaches to assessing motor encoding in the striatum.

To evaluate both hypotheses in mice during unconstrained behavior, mice were recorded during an open field task. The task was chosen because it lacks experimenter-imposed structure or cues, which means the mice were free to perform movements that were unpredictable and heterogeneous. To reduce the variability of movements whose speeds were assessed, we only analyzed periods when the animals were walking (defined as "locomotion"; Fig. 1D,E). While other methods categorized movements into behavioral syllables or distinct subactions (Klaus et al., 2017; Markowitz et al., 2018; Meng et al., 2018), we focused on speed of locomotion in this study. This permitted us to survey the continuous encoding of speed across a single behavioral category (e.g., locomotion) versus specific types of movements which may not occur at multiple speeds (e.g., darting, digging). 
A

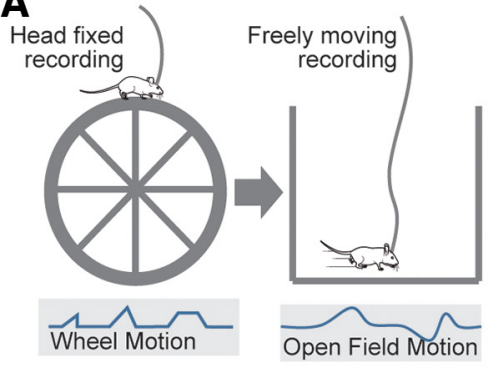

B

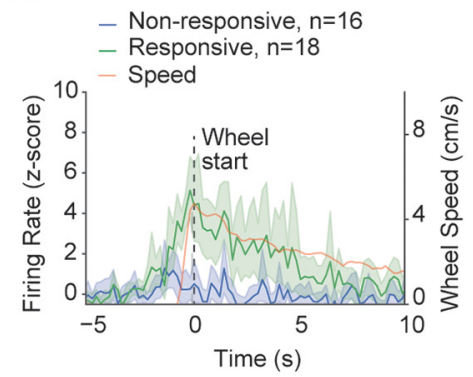

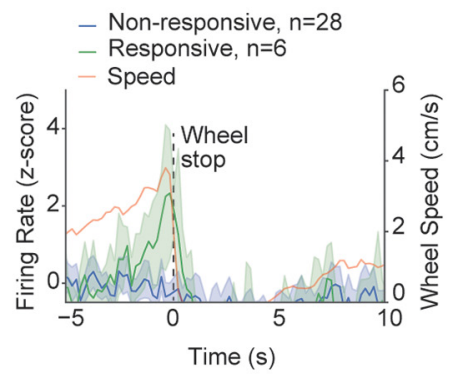

C
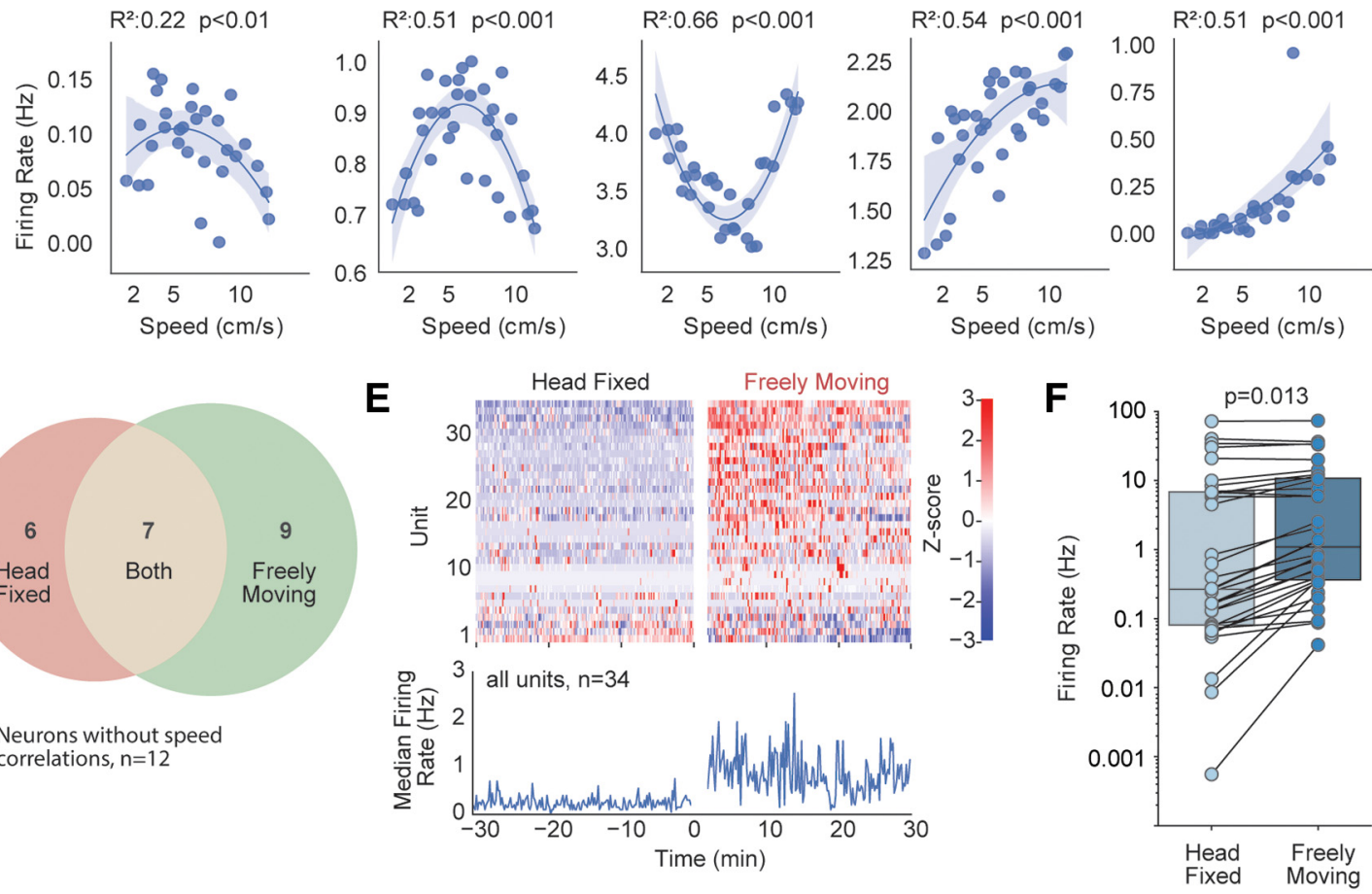

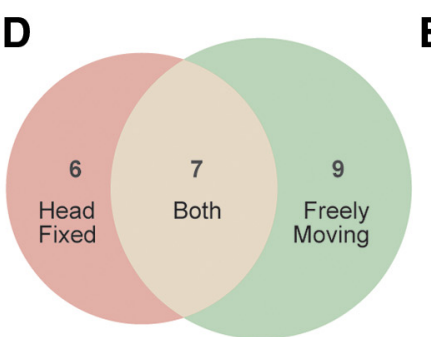

Neurons without speed correlations, $n=12$
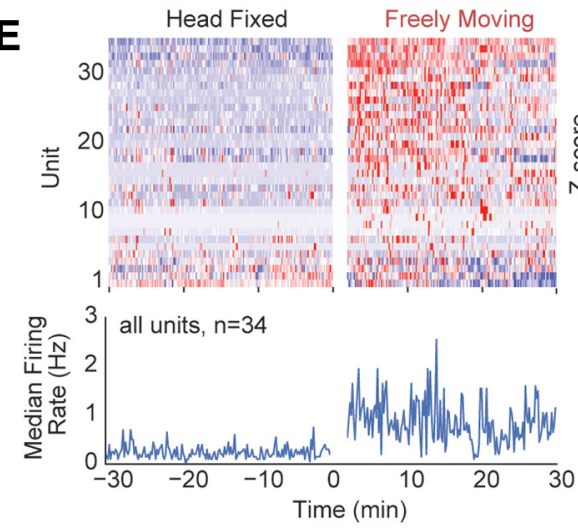

Freely Moving
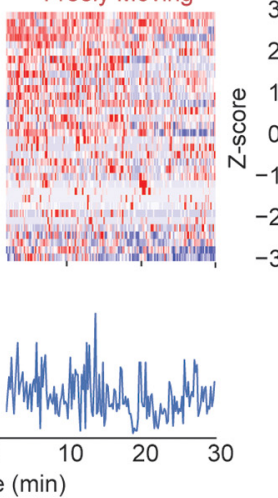

Figure 6. Comparison of speed tuning during head-fixed and free-moving locomotion. $A$, Schematic of experimental setup. A total of 34 units were recorded in 4 mice while they were head-fixed on a wheel for 30-60 min followed by 60 min of free movement in an open field. $\boldsymbol{B}$, Average firing rates and speed for striatal units which do and do not exhibit significant modulation near locomotion starts and stops on the wheel. Shaded regions represent $\pm 95 \% \mathrm{Cl}$. C, Five example striatal units with significant quadratic relationships between firing rate and speed (speed tuning curves) recorded while mice were on the wheel. Shaded regions represent $\pm 95 \% \mathrm{Cl}$. $\boldsymbol{D}$, Venn diagram showing the overlap between the number of striatal units exhibiting speed tuning during the head-fixed and free-moving portions of the session. $\boldsymbol{E}$, Heatmap of firing rate of all recorded units for 30 min on head-fixed wheel followed by 30 min of open-field exploration (top), and median firing rate across these conditions (bottom). $\boldsymbol{F}$, Average firing rates of all recorded striatal units during the head-fixed and free-moving portions of the session.

Fitting our data with linear and quadratic regressions uncovered surprising relationships between individual spiking and locomotion: although several previous recordings in rodent dorsal striatum have reported correlations between firing rates of individual striatal units and speed (Costa et al., 2004; Kim et al., 2014; Panigrahi et al., 2015; Rueda-Orozco and Robbe, 2015; Barbera et al., 2016; Sales-Carbonell et al., 2018), or population activity and speed (Barbera et al., 2016; Bartholomew et al., 2016; Parker et al., 2018), only one reported negative linear correlations (SalesCarbonell et al., 2018) and one preprint examined both linear and nonlinear relationships between movement speed and dorsolateral striatal firing (Taouali et al., 2017). The fact that we observe many units that fire maximally during low-speed locomotion may be attributable to methodological differences between studies. We were able to sample volitional activity continuously and in an unbiased manner by using open-field behavior, whereas prior reports either focused only on active periods triggered by motivation or sensory cues (Costa et al., 2004; Kim et al., 2014; Panigrahi et al., 2015; Rueda-Orozco and Robbe, 2015; Sales-Carbonell et al., 2018); restricted correlations to peak locomotor speeds (Kim et al., 2014; Panigrahi et al., 2015); or recorded over a smaller range of locomotor speeds (Barbera et al., 2016; Parker et al., 2018). Therefore, these studies may have been biased toward specific ranges of movement speed, as determined by the task. Finally, the presence of the nonlinear speed tuning was not attributable to our specific behavioral task, as striatal units recorded from mice constrained on a wheel also exhibited speed tuning (Fig. 6C,D).

While the continuous encoding was present in a larger proportion of units, we also observed evidence of discrete encoding. Twenty-nine percent (61 of 211) of units displayed average firing rates that were modulated near starts and ends of locomotion, but most of those units $(63 \%, 38$ of 61$)$ had firing that also correlated with continuous speed. Thus, perhaps previous reports that only focused on discrete encoding overrepresented or misidentified some striatal neurons as signaling discrete motor change rather than continuous motor parameters. However, given that we also see a smaller percentage $(10 \%, 23$ of 61$)$ that only display the phasic modulation without speed tuning, it also likely true that some neurons signal changes in motor state and/or are tuned to other kinematic parameters. This is a similar argument to that which 
Robbe and colleagues have previously asserted in their characterization of continuous variables during a complex behavior (Rueda-Orozco and Robbe, 2015; Sales-Carbonell et al., 2018). Finally, there also may be regional differences in speed correlations, as our recordings were performed in the dorsomedial striatum, whereas other studies were done in dorsolateral or ventral striatum.

Consistent with previous reports, we observed higher average population firing during locomotion compared with rest (Shi et al., 2004; Yamin et al., 2013; Barbera et al., 2016; Parker et al., 2018). However, the average population firing rate did not increase monotonically with increasing speed (Fig. $3 D, E$ ). Average firing rates, and population calcium activity of iMSNs or dMSNs, increased between $0-1 \mathrm{~cm} / \mathrm{s}$ and $1-2 \mathrm{~cm} / \mathrm{s}$ but largely plateaued $>2 \mathrm{~cm} / \mathrm{s}$ (Figs. $3 D, E, 4 D, E$ ). Similar plateaus are apparent in other reports (Barbera et al., 2016; Parker et al., 2018), but their significance was not explored. Here, we show that this plateau is caused by the summation of diverse tuning curves in individual striatal units, many of which were tuned to low and moderate speed locomotion (Fig. $3 A, B$ ). The distinct tuning profiles reveal diverse continuous encoding at the single-neuron level and suggest that there may be ensembles that support motor behavior in a speed-dependent manner, which may provide contextual guidance for specific actions or behavioral syllables (Kravitz and Peoples, 2008; Klaus et al., 2017; Markowitz et al., 2018).

To explore whether such a population code could be used by downstream structures to infer locomotor speed, we assessed the decoding accuracy of multiple readouts of striatal activity. We found that the decoding accuracy of a multilinear regression analysis performed similarly to machine learning approaches used by others in the literature (Barbera et al., 2016; Parker et al., 2018; Gritton et al., 2019). Our approach differed from previous approaches in several ways. First, we did not predict speed within interleaved periods of held-out data but instead predicted speed from testing data that were recorded after the training data. For certain applications, this approach is beneficial, as it can predict speed on novel data in real time. Second, the relatively simple multilinear regression approach lends itself to rapid decoding without requiring large computational overhead, which can again be useful for real-time analyses. Finally, we explored a range of decoding bin sizes and found that striatal population codes were generally not accurate for predicting speed with single-second resolution but did better at predicting speed across longer timescales (10s of seconds). This may have an important implication for applications that attempt to decode speed from striatal spike trains. Our multiple regression analyses reinforce the idea that downstream structures likely rely on a population code, composed of both subpopulations, that retains spike timing and rate information (Markowitz et al., 2018; Meng et al., 2018).

Despite its involvement in motor control, it remains unclear how the striatum selects actions and governs the speed of these actions. Here, we evaluated two prominent models for motor encoding in the striatum and observed evidence in support of a population code which represents context and speed of locomotion with continuous changes in firing rate.

\section{References}

Barbera G, Liang B, Zhang L, Gerfen CR, Culurciello E, Chen R, Li Y, Lin DT (2016) Spatially compact neural clusters in the dorsal striatum encode locomotion relevant information. Neuron 92:202-213.

Bariselli S, Fobbs WC, Creed MC, Kravitz AV (2019) A competitive model for striatal action selection. Brain Res 1713:70-79.

Barnes TD, Kubota Y, Hu D, Jin DZ, Graybiel AM (2005) Activity of striatal neurons reflects dynamic encoding and recoding of procedural memories. Nature 437:1158-1161.

Bartholomew RA, Li H, Gaidis EJ, Stackmann M, Shoemaker CT, Rossi MA, Yin HH (2016) Striatonigral control of movement velocity in mice. Eur J Neurosci 43:1097-1110.

Berke JD, Okatan M, Skurski J, Eichenbaum HB (2004) Oscillatory entrainment of striatal neurons in freely moving rats. Neuron 43:883-896.

Carelli RM, West MO (1991) Representation of the body by single neurons in the dorsolateral striatum of the awake, unrestrained rat. J Comp Neurol 309:231-249.

Coffey KR, Nader M, West MO (2016) Single body parts are processed by individual neurons in the mouse dorsolateral striatum. Brain Res 1636: 200-207.

Costa RM, Cohen D, Nicolelis MA (2004) Differential corticostriatal plasticity during fast and slow motor skill learning in mice. Curr Biol 14:1124-1134.

Cui G, Jun SB, Jin X, Pham MD, Vogel SS, Lovinger DM, Costa RM (2013) Concurrent activation of striatal direct and indirect pathways during action initiation. Nature 494:238-242.

Franklin K, Paxinos G (2019) Paxinos and Franklin's the mouse brain in stereotaxic coordinates. Compact-5th Edition. Academic Press.

Geddes CE, Li H, Jin X (2018) Optogenetic editing reveals the hierarchical organization of learned action sequences. Cell 174:32-43.e15.

Gong S, Doughty M, Harbaugh CR, Cummins A, Hatten ME, Heintz N, Gerfen CR (2007) Targeting cre recombinase to specific neuron populations with bacterial artificial chromosome constructs. J Neurosci 27:98179823.

Gritton HJ, Howe WM, Romano MF, DiFeliceantonio AG, Kramer MA, Saligrama V, Bucklin ME, Zemel D, Han X (2019) Unique contributions of parvalbumin and cholinergic interneurons in organizing striatal networks during movement. Nat Neurosci 22:586-597.

Isomura Y, Takekawa T, Harukuni R, Handa T, Aizawa H, Takada M, Fukai T (2013) Reward-modulated motor information in identified striatum neurons. J Neurosci 33:10209-10220.

Jin X, Costa RM (2010) Start/stop signals emerge in nigrostriatal circuits during sequence learning. Nature 466:457-462.

Jog MS, Kubota Y, Connolly CI, Hillegaart V, Graybiel AM (1999) Building neural representations of habits. Science 286:1745-1749.

Kim N, Barter JW, Sukharnikova T, Yin HH (2014) Striatal firing rate reflects head movement velocity. Eur J Neurosci 40:3481-3490.

Klaus A, Martins GJ, Paixao VB, Zhou P, Paninski L, Costa RM (2017) The spatiotemporal organization of the striatum encodes action space. Neuron 95:1171-1180.e7.

Kravitz AV, Peoples LL (2008) Background firing rates of orbitofrontal neurons reflect specific characteristics of operant sessions and modulate phasic responses to reward-associated cues and behavior. J Neurosci 28:1009-1018.

Kravitz AV, Freeze BS, Parker PR, Kay K, Thwin MT, Deisseroth K, Kreitzer AC (2010) Regulation of parkinsonian motor behaviours by optogenetic control of basal ganglia circuitry. Nature 466:622-626.

Kulik JM, Pawlak AP, Kalkat M, Coffey KR, West MO (2017) Representation of the body in the lateral striatum of the freely moving rat: fast spiking interneurons respond to stimulation of individual body parts. Brain Res 1657:101-108.

Lemos JC, Friend DM, Kaplan AR, Shin JH, Rubinstein M, Kravitz AV, Alvarez VA (2016) Enhanced GABA transmission drives bradykinesia following loss of dopamine D2 receptor signaling. Neuron 90:824-838.

Markowitz JE, Gillis WF, Beron CC, Neufeld SQ, Robertson K, Bhagat ND, Peterson RE, Peterson E, Hyun M, Linderman SW, Sabatini BL, Datta SR (2018) The striatum organizes 3D behavior via moment-to-moment action selection. Cell 174:44-58.e17.

Martiros N, Burgess AA, Graybiel AM (2018) Inversely active striatal projection neurons and interneurons selectively delimit useful behavioral sequences. Curr Biol 28:560-573.e5.

Meng C, Zhou J, Papaneri A, Peddada T, Xu K, Cui G (2018) Spectrally resolved fiber photometry for multi-component analysis of brain circuits. Neuron 98:707-717.e4.

Mink JW (1996) The basal ganglia: focused selection and inhibition of competing motor programs. Prog Neurobiol 50:381-425.

Moran DW, Schwartz AB (1999) Motor cortical representation of speed and direction during reaching. J Neurophysiol 82:2676-2692.

Panigrahi B, Martin KA, Li Y, Graves AR, Vollmer A, Olson L, Mensh BD, Karpova AY, Dudman JT (2015) Dopamine is required for the neural representation and control of movement vigor. Cell 162:1418-1430. 
Parker JG, Marshall JD, Ahanonu B, Wu YW, Kim TH, Grewe BF, Zhang Y, Li JZ, Ding JB, Ehlers MD, Schnitzer MJ (2018) Diametric neural ensemble dynamics in parkinsonian and dyskinetic states. Nature 557:177-182.

Puryear CB, Kim MJ, Mizumori SJ (2010) Conjunctive encoding of movement and reward by ventral tegmental area neurons in the freely navigating rodent. Behav Neurosci 124:234-247.

Robbe D (2018) To move or to sense? Incorporating somatosensory representation into striatal functions. Curr Opin Neurobiol 52:123-130.

Rueda-Orozco PE, Robbe D (2015) The striatum multiplexes contextual and kinematic information to constrain motor habits execution. Nat Neurosci 18:453-460.

Sales-Carbonell C, Taouali W, Khalki L, Pasquet MO, Petit LF, Moreau T, Rueda-Orozco PE, Robbe D (2018) No discrete start/stop signals in the dorsal striatum of mice performing a learned action. Curr Biol 28:30443055.e5.
Schultz W, Tremblay L, Hollerman JR (2003) Changes in behavior-related neuronal activity in the striatum during learning. Trends Neurosci 26:321-328

Shi LH, Luo F, Woodward DJ, Chang JY (2004) Neural responses in multiple basal ganglia regions during spontaneous and treadmill locomotion tasks in rats. Exp Brain Res 157:303-314.

Taouali W, Rueda-Orozco PE, Robbe D (2017) A minority-ruled population coding of kinematics in the striatum. BioRxiv 130237, Version 2. doi: 10.1101/130237.

Turner RS, Desmurget M (2010) Basal ganglia contributions to motor control: a vigorous tutor. Curr Opin Neurobiol 20:704-716.

Yamin HG, Stern EA, Cohen D (2013) Parallel processing of environmental recognition and locomotion in the mouse striatum. J Neurosci 33:473484.

Yttri EA, Dudman JT (2016) Opponent and bidirectional control of movement velocity in the basal ganglia. Nature 533:402-406. 